\title{
Reciprocal Polynomials Having Small Measure. II
}

\author{
By David W. Boyd*
}

\begin{abstract}
We consider here polynomials with integer coefficients having measure at most 1.3. In the first paper of this series we determined all such polynomials of degree at most 16 , and all such polynomials of height 1 and degree at most 26 . In this paper we extend these results to all polynomials of degree at most 20 , and all polynomials of height 1 and degree at most 32 . We observe some curious statistics concerning the number of roots outside the unit circle for the polynomials investigated here.
\end{abstract}

1. Introduction. Recall, from [1], that the measure $M(P)$ of the polynomial $P(x)=a_{0} x^{n}+\cdots+a_{n}$ with $a_{0} \neq 0$ and zeros $\alpha_{1}, \ldots, \alpha_{n}$ is defined by

$$
M(P)=\left|a_{0}\right| \prod_{i=1}^{n} \max \left(\left|\alpha_{i}\right|, 1\right)
$$

A polynomial $P$ of degree $n$ is reciprocal if $P(x)=x^{n} P\left(x^{-1}\right)$.

We are interested in the possible values of $M(P)$ as $P$ varies over all reciprocal polynomials with integer coefficients. If $P$ is cyclotomic then $M(P)=1$. The smallest known value of $M(P)>1$ was found in 1933 by D. H. Lehmer [2], namely

$$
M\left(x^{10}+x^{9}-x^{7}-x^{6}-x^{5}-x^{4}-x^{3}+x+1\right)=1.1762808183 \ldots
$$

In [1], we developed an algorithm capable of surveying large sets of polynomials and used it to find all reciprocal $P$ with $\operatorname{deg}(P) \leq 16$ and $M(P) \leq 1.3$, and also all reciprocal $P$ with $\operatorname{deg}(P) \leq 26, H(P)=1$ and $M(P) \leq 1.3$, where $H(P)=\max \left|a_{i}\right|$ is the height of $P$.

In this paper we use the same algorithm to extend the results of [1] to all such $P$ with $\operatorname{deg}(P) \leq 20$ and all $P$ with $\operatorname{deg}(P) \leq 32$ and $H(P)=1$. No $P$ with a smaller measure than Lehmer's example was discovered.

Indeed, the tables of $P$ with $\operatorname{deg}(P) \leq 20$ published in [1] were found to be complete. That is, all $P$ with $\operatorname{deg}(P) \leq 20$ and $M(P) \leq 1.3$ divide a $Q$ with $\operatorname{deg}(Q) \leq \operatorname{deg}(P)+4, H(Q)=1$ and $M(Q) \leq 1.3$. This suggests that the lists presented here are probably complete up to $\operatorname{deg}(P) \leq 26$.

The opportunity to extend the computations of [1] arose because of a recent policy of the University of British Columbia Computing Centre to sell idle time on its Amdahl 5860 at reduced rates. In addition, this machine is approximately three times faster than the Amdahl 470 on which the computations of [1] were performed. The complete enumeration for $\operatorname{deg}(P)=20$ took 6.5 hours of CPU time.

Received May 20, 1988.

1980 Mathematics Subject Classification (1985 Revision). Primary 12-04; Secondary 11 Y 40.

*Supported in part by an NSERC operating grant. 
2. Discussion of the Results. Call $P$ primitive if it is not of the form $Q\left(x^{s}\right)$, $s \geq 2$. The appendix in the Supplements section of this issue lists one of $P(x)$ or $P(-x)$ for all primitive irreducible polynomials with $1<M(P)<1.3$ and $\operatorname{deg}(P)=n$ for $18 \leq n \leq 32$, under the restriction that if $22 \leq n \leq 32$ then $P$ divides a polynomial $Q$ with $\operatorname{deg}(Q) \leq 32, H(Q)=1$ and $M(Q)<1.3$. For $n \leq 16$, see [1].

Table 1 lists the smallest known values of $M(P)$ for reciprocal $P$ with $\operatorname{deg}(P)=$ $n, 4 \leq n \leq 40$. For $n \leq 20$, these are the minimum possible. For $22 \leq n \leq 32$, $M(P)$ is the minimum attained by a $P$ which divides a $Q$ with $\operatorname{deg}(Q) \leq 32$, $H(Q)=1$ and $M(Q)<1.3$. The examples for $34 \leq n \leq 40$ come from certain families of $P$ known to have small measure [1, Section 5].

TABLE 1

Smallest known values of $M(P)>1$ for reciprocal $P$ of fixed degree. $\nu(P)$ is the number of zeros of $P$ outside the unit circle.

For $\operatorname{deg}(P)$ at most 20 , these are $\min M(P)$

\begin{tabular}{|c|c|c|c|c|c|c|c|c|c|c|c|c|c|c|c|c|c|c|c|c|c|}
\hline $\operatorname{deg}$ & measure & $\nu$ & \multicolumn{19}{|c|}{ first half of polynomial } \\
\hline 4 & 1.7220838057 & 1 & 1 & & -1 & & & & & & & & & & & & & & & & \\
\hline 6 & 1.4012683679 & 1 & 1 & 0 & -1 & 1 & & & & & & & & & & & & & & & \\
\hline 8 & 1.2806381563 & 1 & 1 & 0 & 0 & 1 & -1 & & & & & & & & & & & & & & \\
\hline 10 & 1.1762808183 & 1 & 1 & 1 & 0 & -1 & -1 & -1 & & & & & & & & & & & & & \\
\hline 12 & 1.2277855587 & 2 & 1 & 1 & 1 & 0 & -1 & -1 & -1 & & & & & & & & & & & & \\
\hline 14 & 1.2000265240 & 1 & 1 & 0 & 0 & 1 & -1 & 0 & & -1 & & & & & & & & & & & \\
\hline 16 & 1.2242789072 & 2 & 1 & 1 & 0 & -1 & -1 & 0 & 1 & 1 & 1 & & & & & & & & & & \\
\hline 18 & 1.1883681475 & 1 & 1 & 1 & 1 & 1 & 0 & 0 . & -1 & -1 & $-1-1$ & & & & & & & & & & \\
\hline 20 & 1.2128241810 & 2 & 1 & 1 & 0 & 0 & 1 & 1 & 0 & -1 & $-1-1$ & -1 & & & & & & & & & \\
\hline 22 & 1.2050198542 & 2 & 1 & 0 & 1 & 0 & 0 & 1 & -1 & 1 & 00 & 1 & -1 & & & & & & & & \\
\hline 24 & 1.2188551503 & 2 & 1 & 0 & 0 & 0 & 0 & 1 & 0 & -1 & $0 \quad 0$ & 0 & 0 & -1 & & & & & & & \\
\hline 26 & 1.2237774549 & 3 & 1 & 1 & 1 & 0 & 0 & -1 & -1 & -1 & -1 & 0 & 1 & 0 & 1 & & & & & & \\
\hline 28 & 1.2079500284 & 2 & 1 & 1 & 1 & 1 & 0 & 0 & 0 & -1 & $-1-1$ & -1 & 0 & 0 & 0 & 1 & & & & & \\
\hline 30 & 1.2256198520 & 2 & 1 & 0 & 1 & 0 & 0 & 0 & 0 & 0 & 00 & 0 & 0 & 0 & 1 & 0 & 1 & & & & \\
\hline 32 & 1.2361984699 & 3 & 1 & 0 & $\mathbf{0}$ & 0 & 0 & 0 & 0 & 1 & 0 & 0 & 0 & 0 & 0 & 0 & 0 & 1 & & & \\
\hline 34 & 1.2299990397 & 3 & 1 & 0 & 0 & 0 & 0 & 0 & -1 & 0 & 0 & 0 & -1 & 0 & 0 & 0 & 0 & 0 & 1 & & \\
\hline 36 & 1.2294828102 & 4 & 1 & 1 & 1 & 0 & 0 & -1 & -1 & -1 & 0 & 1 & 1 & 0 & -1 & -2 & -1 & 0 & 1 & & \\
\hline 38 & 1.2234473814 & 3 & 1 & 1 & 0 & -1 & -1 & 0 & 0 & -1 & -1 & 1 & 1 & 0 & 0 & 1 & 1 & 0 & $-1-1$ & -1 & \\
\hline 40 & 1.2362495573 & 3 & 1 & 0 & 0 & 1 & 0 & 0 & 0 & 0 & 0 & 0 & 0 & 0 & 0 & 0 & 0 & 0 & 10 & 0 & 1 \\
\hline
\end{tabular}

Table 2 classifies the known $P$ with $1<M(P)<1.3$ according to $\operatorname{deg}(P)$ and $\nu(P)$, the number of roots of $P$ outside the unit circle. The results show a remarkable deviation from uniformity: if $\operatorname{deg}(P) \equiv 0(\bmod 4)$, then even values of $\nu(P)$ predominate, while if $\operatorname{deg}(P) \equiv 2(\bmod 4)$, odd values of $\nu(P)$ predominate. The modal value of $\nu(P)$ for fixed $\operatorname{deg}(P)$ is strikingly large for $\operatorname{deg}(P) \geq 20$. If imprimitive polynomials are admitted, then the results are even more striking; for example, if $\operatorname{deg}(P)=32$, then polynomials of the form $Q\left(x^{2}\right), \operatorname{deg}(Q)=16$ and $Q\left(x^{4}\right), \operatorname{deg}(Q)=8$ increase the number of $P$ with $\nu(P)=4$ to $26+12+1=39$ out of a total of 69 . 
It would be of considerable interest to give an explanation of the phenomenon. Perhaps it is connected with the existence of the families of $P$ alluded to above.

We repeat a remark from [1] concerning the computations with polynomials of height 1 . Siegel's lemma can be used to show that each $P$ with $M(P)<2$ must divide a $Q$ with $H(Q)=1$. However, it is not known whether one can also insure that $M(Q)=M(P)$ so that $Q / P$ is cyclotomic. It would be of interest to decide whether or not this is the case and to give realistic bounds on $\operatorname{deg}(Q)-\operatorname{deg}(P)$. We know of no example which requires $\operatorname{deg}(Q)>\operatorname{deg}(P)+4$, but presumably there are examples with $\operatorname{deg}(Q)-\operatorname{deg}(P)$ arbitrarily large.

\section{TABLE 2}

Known primitive irreducible polynomials with measures satisfying $1<M(P)<1.3$, classified according to $n=\operatorname{deg}(P)$ and $\nu=\nu(P)$, the number of roots of $P$ outside the unit circle. (Data complete to $n=20$. Missing entries are 0 ).

\begin{tabular}{rrrrrrrrrr}
\hline$n \backslash$ & $\nu=1$ & 2 & 3 & 4 & 5 & 6 & total & odd & even \\
\hline 8 & 1 & & & & & & 1 & 1 & 0 \\
10 & 5 & 2 & & & & & 7 & 5 & 2 \\
12 & 1 & 4 & & & & & 5 & 1 & 4 \\
14 & 4 & 5 & 2 & & & & 11 & 6 & 5 \\
16 & 1 & 12 & 0 & 1 & & & 14 & 1 & 13 \\
18 & 7 & 8 & 8 & 0 & & & 23 & 15 & 8 \\
20 & 4 & 22 & 3 & 6 & & & 35 & 7 & 28 \\
22 & 4 & 12 & 27 & 4 & 1 & & 48 & 32 & 16 \\
24 & 2 & 29 & 3 & 11 & 0 & 1 & 46 & 5 & 41 \\
26 & 7 & 11 & 21 & 11 & 11 & 0 & 61 & 39 & 22 \\
28 & 1 & 15 & 14 & 28 & 1 & 0 & 59 & 16 & 43 \\
30 & 4 & 9 & 33 & 18 & 8 & 1 & 73 & 45 & 27 \\
32 & 0 & 9 & 7 & 26 & 7 & 5 & 54 & 14 & 40 \\
\hline
\end{tabular}

Department of Mathematics

University of British Columbia

Vancouver, B.C., Canada V6T 1Y4

E-mail: userboyz@ubcmtsg. bitnet

1. D. W. BOYD, "Reciprocal polynomials having small measure," Math. Comp., v. 35, 1980, pp. 1361-1377.

2. D. H. LEHMER, "Factorization of certain cyclotomic functions", Ann. of Math. (2), v. 34, 1933, pp. 461-479. 


\title{
Supplement to Reciprocal Polynomials Having Small Measure. II
}

\author{
By David W. Boyd
}

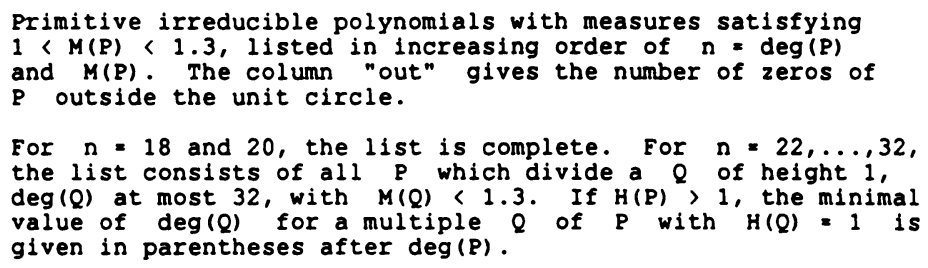

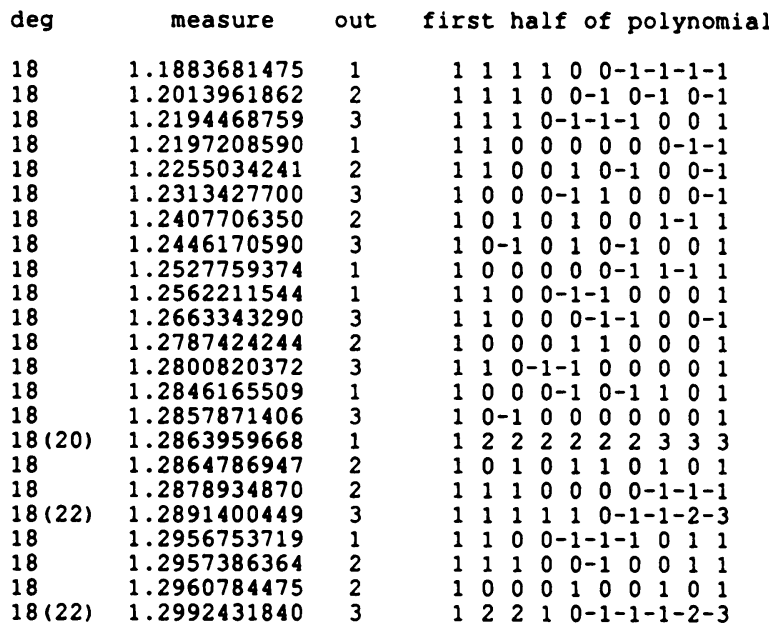




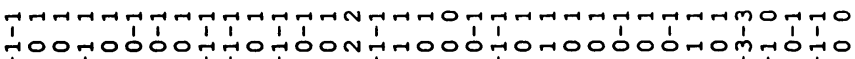

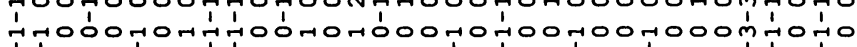

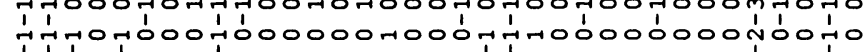

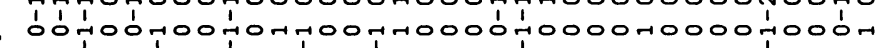
O 0 H.

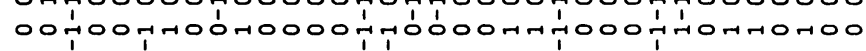
Há,

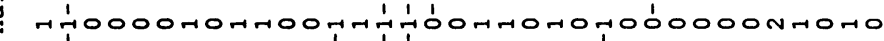

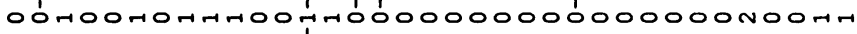

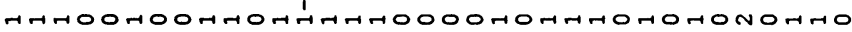

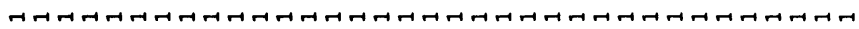

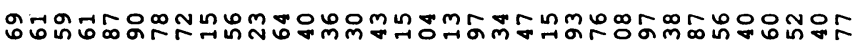

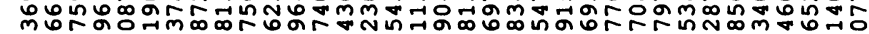

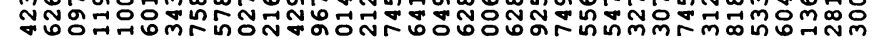

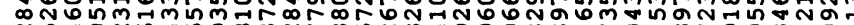

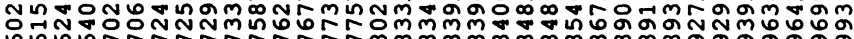

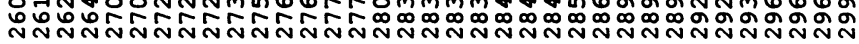

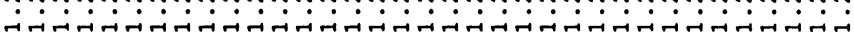

$$
\stackrel{\sim}{\sim}
$$

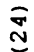

व

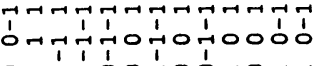

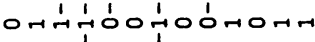

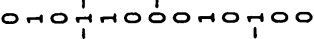

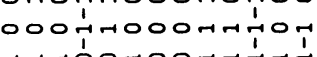

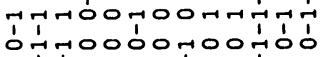

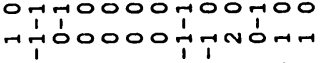

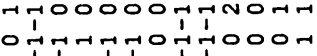

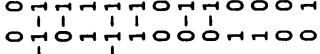

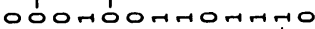

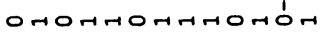

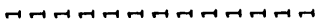

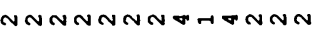

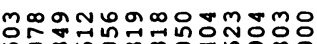

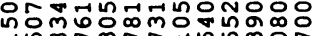

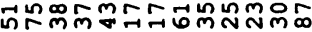

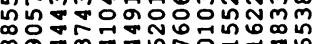

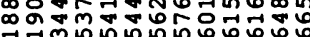

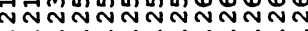

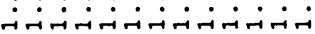

$\stackrel{\infty}{\stackrel{\infty}{\sim}}$

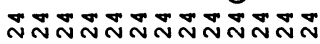

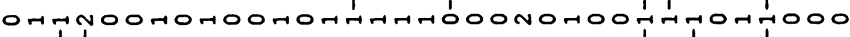
Oन

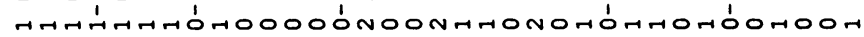

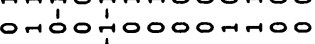

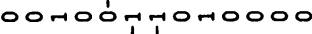
-

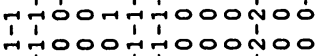
$0 \frac{1}{1} 00 \% 000000 \frac{1}{1}$ ०

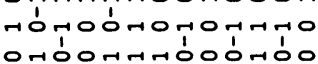

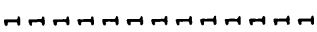

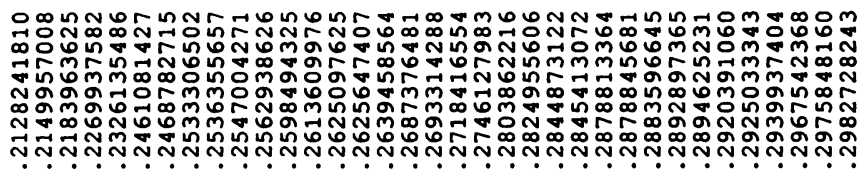

莡

NMTNMNMMNMMNM

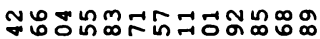

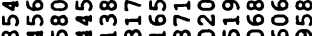

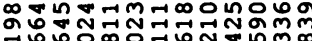

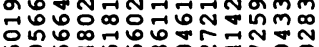
ํํำํำ

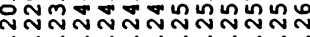

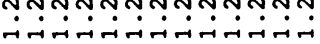


ન-બ્ર

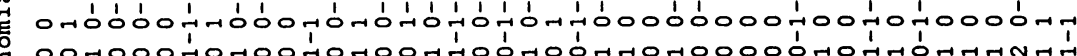

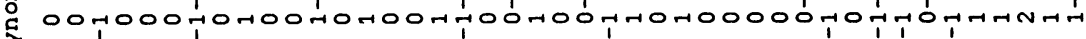
خ 00 O 000 H.

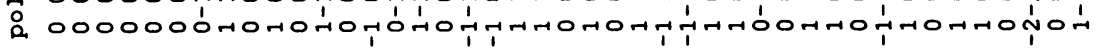

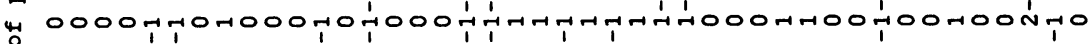

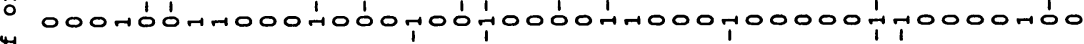

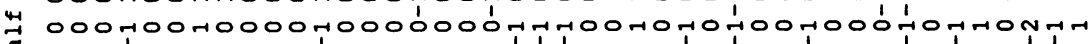

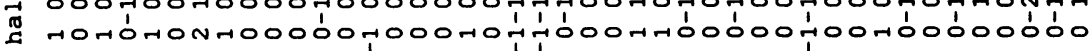

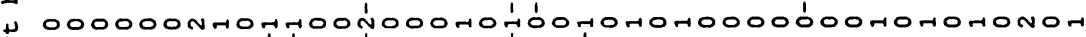

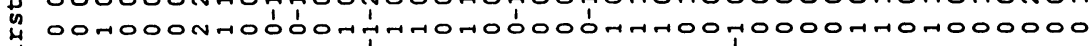

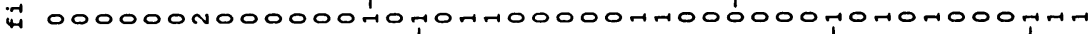

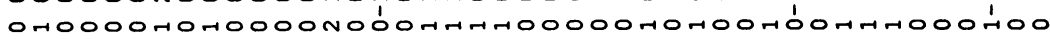
भનनન

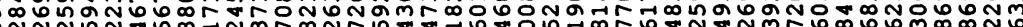

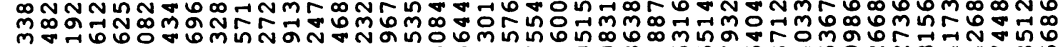

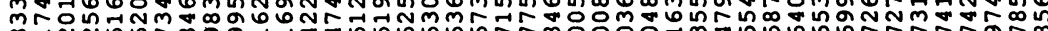

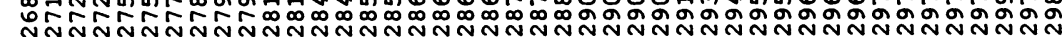

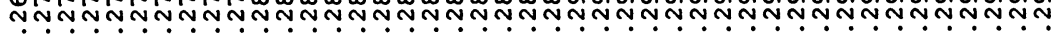

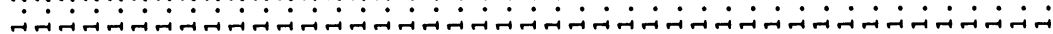

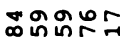
Tr.

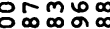

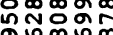

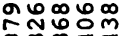

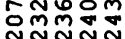
i-i-i-i-i

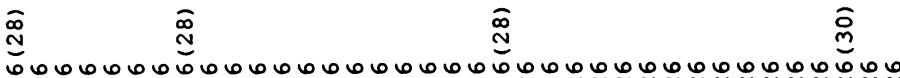

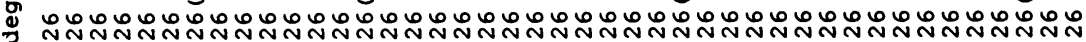

을

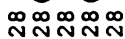

क्

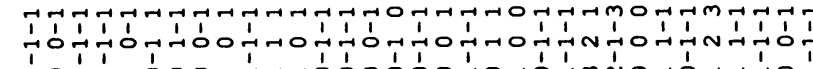
Ė

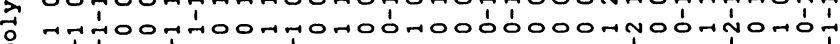

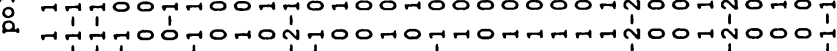

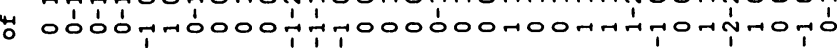

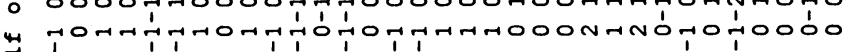

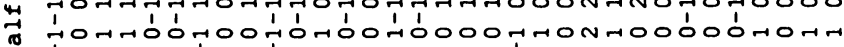

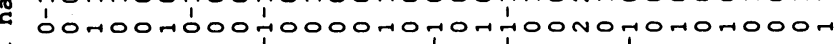

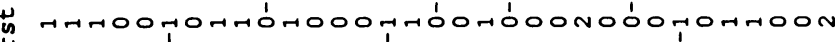

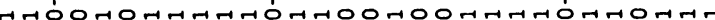

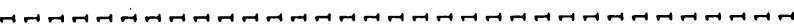

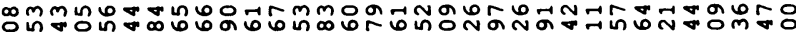

峁

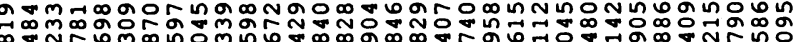

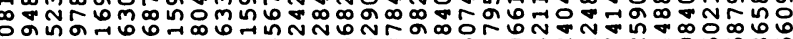

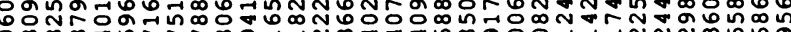

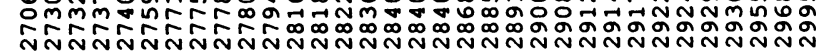

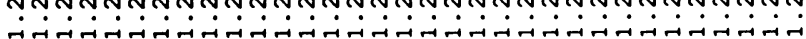

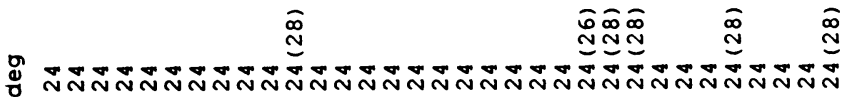

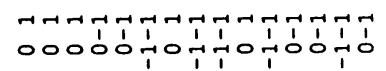

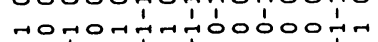
0 O. o0 t०

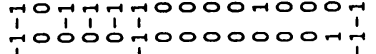

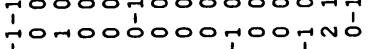
tiontoroootithómo

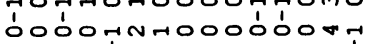

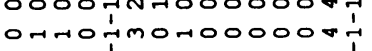
homámmaomoomó modínnohtohthna

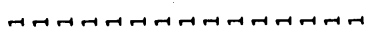

MNMFMNNMMMNNANM

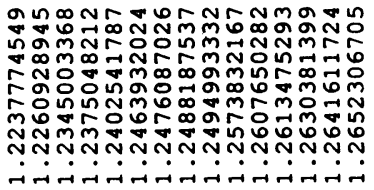
$\stackrel{\infty}{\sim}$ $\stackrel{\infty}{\stackrel{\infty}{0}}$

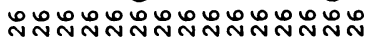




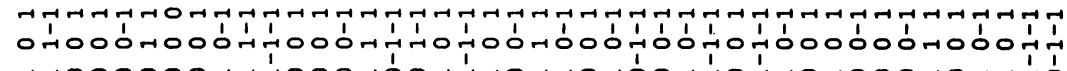

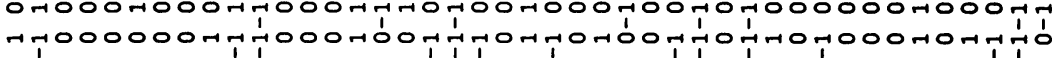

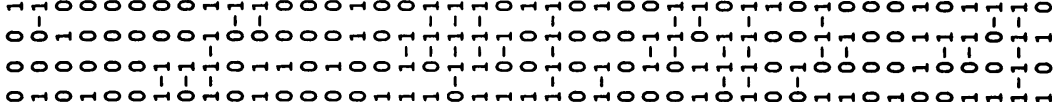

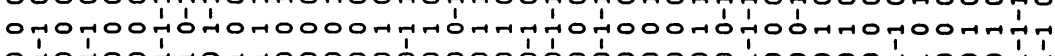

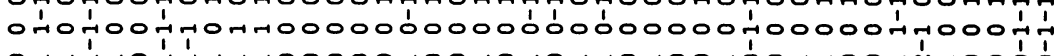
OFHFO I'

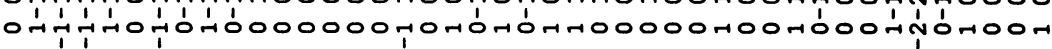

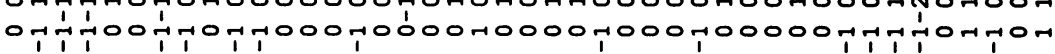

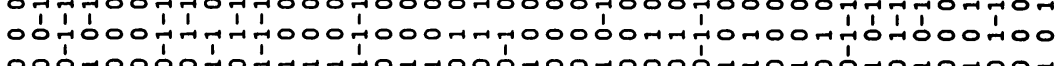

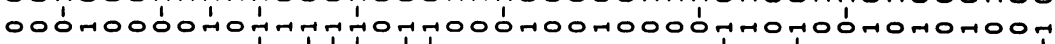

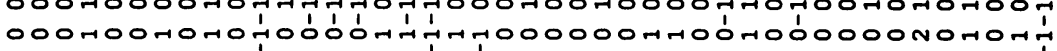

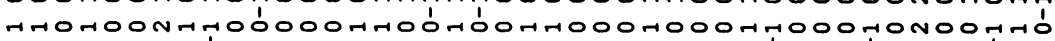

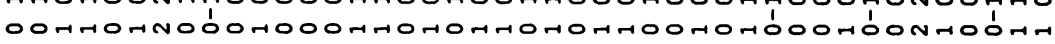

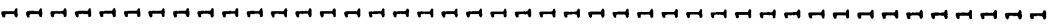

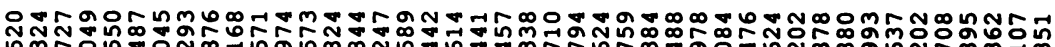

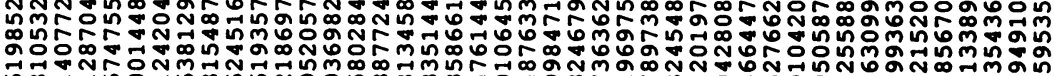

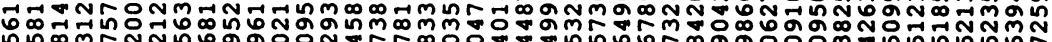

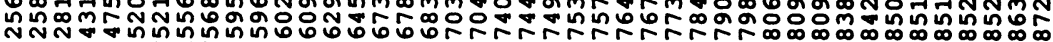

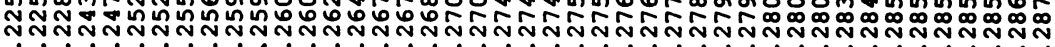

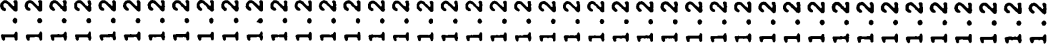

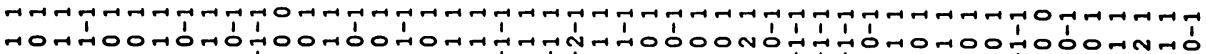

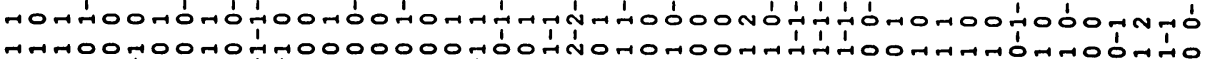

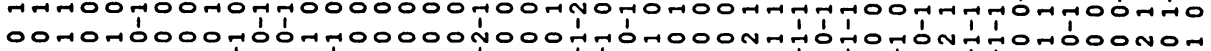

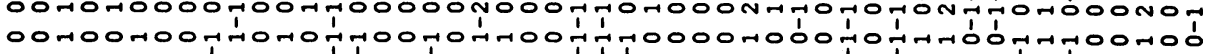

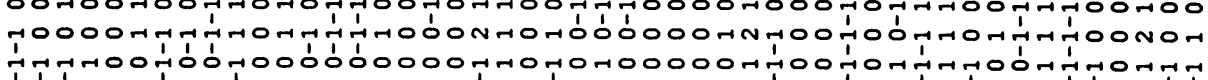
II İ

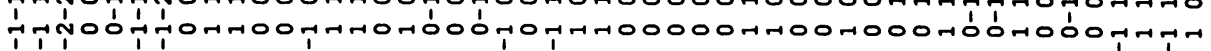
Í ÍnO

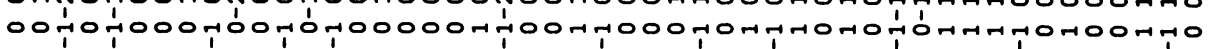

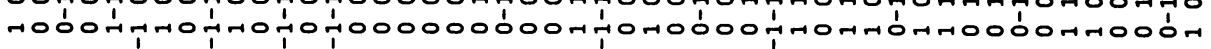

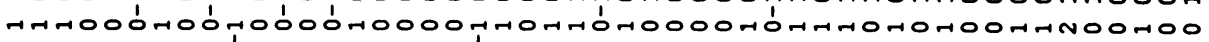

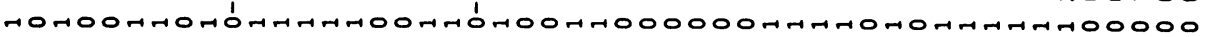

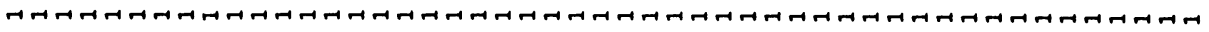

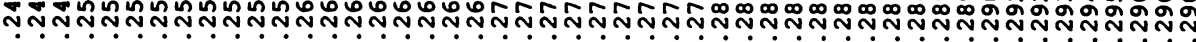

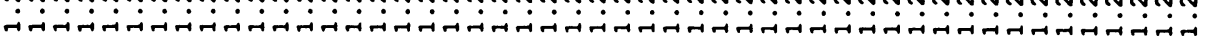

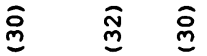

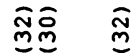
กิ
กิ

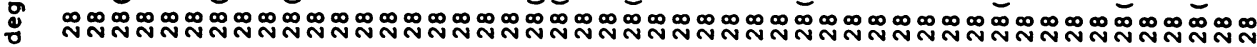




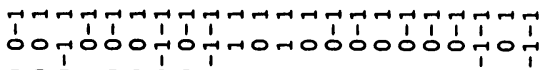

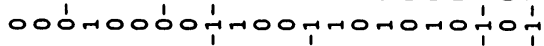

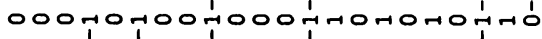

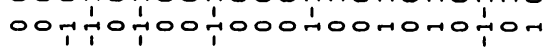

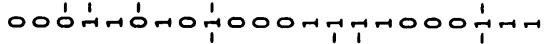
000न00न0ठ́न000 1́,

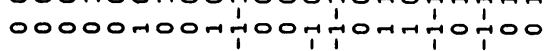

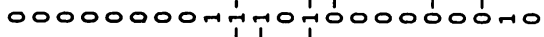

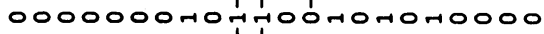

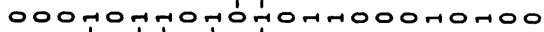

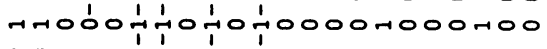

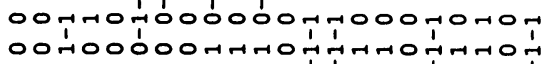
ठ00

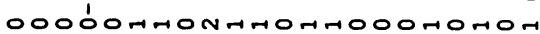

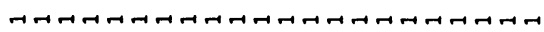

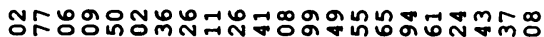

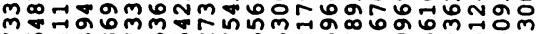
Tำ

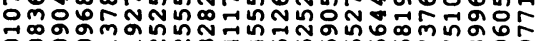
ठㅇㅇㅇำ

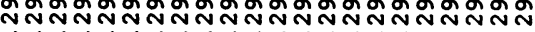

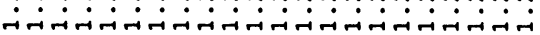

m

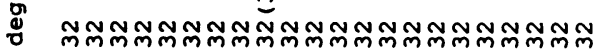

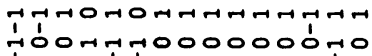
11
1
1

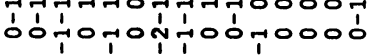

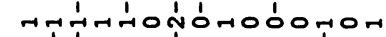

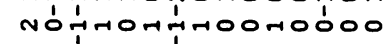
NHó0000́0HA00000 hónhHAh00000000

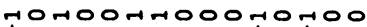
'́ No000न0न00न000

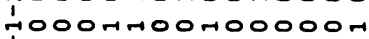
'́00न0न000000000

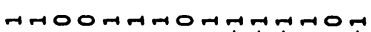
नन

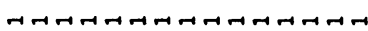

भल

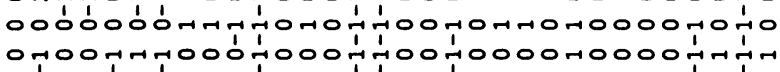

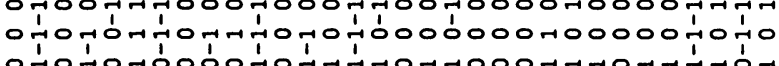
०

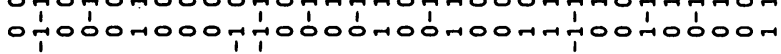
- '1 0 न 0 O ठ ठ́०

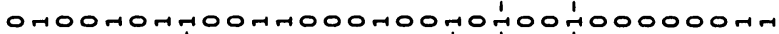

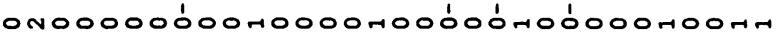

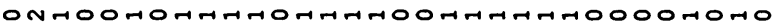
નનનનનનનનનનનનનનનનનનનનનનનનનનનનનનનન

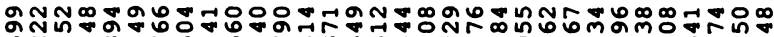

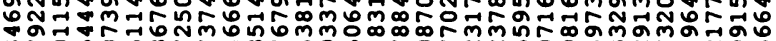

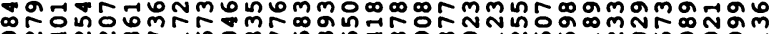

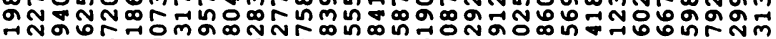

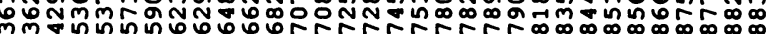

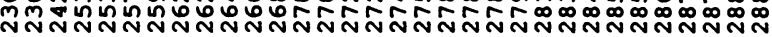
M. 